\title{
Early detection of hymenal anomalies and variants
}

\author{
Paul Merlob • Gil Klinger
}

Received: 6 November 2014 / Accepted: 6 March 2015 /Published online: 17 March 2015

(C) Springer-Verlag Berlin Heidelberg 2015

In a recent article, Watrowski and collaborators described the first case of sub-occlusive hymenal variants (microperforate hymen and septate hymen) in 16-yearold dizygotic twins [4]. The authors emphasized that understanding of the sub-occlusive hymenal variants is crucial for proper evaluation of the female genitalia in children including their possible familial occurrence and the need for surgical intervention.

We performed a prospective study to investigate the various types of neonatal hymens and their influence on physiologic vaginal discharge [3]. A total of 333 female infants were examined after birth. We distinguished seven types of hymenal shapes and orifices and calculated their incidence. Only two hymenal shapes (hymenal band and almost imperforated hymen defined as subocclusive variants by Watrowski et al.) interfere with neonatal vaginal discharge. The possible changes in hymenal configuration during childhood as a result of aging were not studied. We also observed a high incidence of hymenal tags $(5.75 \%)$ and hymenal bands $(2.7 \%)$ during routine physical examination of 974 female in-

Communicated by Patrick Van Reempts

This paper is a correspondence for http://dx.doi.org/10.1007/S00431013-2123-3.

P. Merlob $\cdot$ G. Klinger $(\bowtie)$

Neonatology Department, Schneider Children's Medical Center of Israel, Petah Tiqva, Israel

e-mail: gilk1@post.tau.ac.il

P. Merlob $\cdot$ G. Klinger

Sackler Medical School, Tel Aviv University, Tel Aviv, Israel fants within the first $24 \mathrm{~h}$ of life [2]. A simple test was used to demonstrate the superficial position of the hymenal band and its lack of connection with an internal structure, similarly to that used by Watrowski et al. [4]. Based on our studies [1-3], we believe that most (if not all) of the variants or anomalies of the external genitalia in the female infant can and should be diagnosed at birth. The physical examination of every newborn or infant is not complete without careful inspection of the external genitalia. In this manner, the variants and anomalies of the hymen will be detected early in life and counseling and reassurance of the parents about the benign outcome of many of them or the necessity of early intervention (e.g., imperforated hymen associated with hydrometrocolpos) will be possible.

Author's Contributions The comment was jointly written by Paul Merlob and Gil Klinger.

\section{References}

1. Merlob P, Bahari C, Liban E, Reisner SH (1978) Cysts of the female external genitalia in the newborn infant. Am J Obstet Gynecol 132: 607-610

2. Mor N, Merlob P, Reisner SH (1983) Tags and bands of the female external genitalia in the newborn infant. Clin Pediatr 22:122-124

3. Mor N, Merlob P, Reisner SH (1986) Types of hymen in the newborn infant. Eur J Obstet Gynecol Reprod Biol 22:225-228

4. Watrowski R, Jager C, Gerber M, Klein C (2014) Hymenal anomalies in twins - review of the literature and case report. Eur J Pediatr 173: $1407-1412$ 